Bulgarie, pays essentiellement agricole ne doit pas oublier que toutes les dépenses faites pour l'amélioration des industries animales seront remboursées largement par l'extension de ses marchés extérieurs. Les vétérinaires ont ici à jouer ur rôle dont ils comprennent tous les premiers la haute importance.

\title{
LA VITAMINE C DANS LE LAIT CONCENTRÉ SUCRÉ
}

Par Dr A. BAKKE

Chef des Services scientifiques de la Nestlé et Anglo-Swiss Condensed Milk Co.

Assisté par Mile V. ASCHEHOUG

Assistante au laboratoire de recherches de la Nestlé et Anglo-Swiss Cond. Milk Co.

Parmi les vitamines les plus connues (nous parlons des vitamines A, B, C et D), c'est la vitamine C qui est la plus fragile. On peut dire que toutes les vitamines sont plus thermostables qu'on ne l'avait considéré au début de l'étude de ces substances ou principes. On sait aujourd'hui que les conditions dans lesquelles agit la chaleur, notamment le milieu, et surtout l'oxydation, jouent un rôle essentiel dans la résistance des vitamines à la chaleur. Ceci s'applique également à la durée de leur conservation. Il n'en reste pas moins 'que la vitamine " $\mathrm{C}$ » est la plus fragile de toutes. Il est donc particulièrement intéressant d'examiner comment elle se comporte dans les conserves de lait et surtout dans le lait concentré.

Il y a deux grandes elasses de lait concentré. L'une, comprend uniquement le lait concentré sucré, dont la conservation est assurée uniquement par l'extraction de la plus grande partie de l'eau qui entre dans sa composition et par l'adjonction d'une quantité convenable de saccharose. Le lait con:entré sucré n'est pas stérilisé.

L'autre classe comprend essentiellement les laits stérilisés non sucrés, concentrés ou non, et dont la conservation est assurée par une stérilisation thermique rigoureuse, qui doit, pour atteindre son but, détruire absolument tous les germes contenus dans le lait. sucré.

Le produit qui nous intéresse dans ce travail est le lait concentré

Pour nos essais, nous avons utilisé des cobayes, quoique certains auteurs estiment que la sensibilité des animaux à l'absence de vitamines "C $\mathrm{C}$ est trop grande pour qu'il soit possible d'en tirer des conclusions applicables à l'alimentation humaine.

Cependant, comme il existe des raisons de croire que le rôle de la vitamine " $\mathrm{C}$ " ne se limite pas à une action antiscorbutique, mais agit 
encore d'une façon plus étendue dans l'harmonie de la nutrition, il nous semble indiqué d'utiliser des animaux dont la sensibilité soit maximum. De plus, des essais de ce genre peuvent être faits facilement avec des cobayes, moyennant certaines précautions, dont nous allons parler plus loin.

Il était tout d'abord nécessaire d'établir la quantité de vitamines " $\mathrm{C}$ " devant être présente pour protéger le cobaye contre le scorbut. Ce point avait été éclairci pour le jus d'orange et de citron. En raison du but que nous poursuivions, nous avons préféré déterminer les quantités minima pour le lait de femme et pour le lait de vache frais. Nous avons trouvé qu'environ $60 \mathrm{~cm}^{3}$ de lait humain par $24 \mathrm{~h}$. sont suffisants pour permettre un bon développement des cobayes, sans aucun symptôme scorbutique, et qu'une quantité à peu près identique de lait de vache frais est nézessaire pour obtenir le même résultat. On trouve, dans la littérature, des indications très variables à ce sujet. Cela est évidemment dû en partie à ce que les différents laits naturels ne contiennent pas les mêmes quantités de vitamines " $\mathrm{C}$ ".

Nos très nombreuses séries d'essais nous ont prouvé, cependant, que le facteur le plus important est la quantité de lait qui est réellement absorbée par le sujet soumis à l'expérience.

Le cobaye n'aime pas le lait humain, ni le lait de vache en forte quantité. Très fréquemment, les sujets refusent d'avaler le lait. II faut alors le faire prendre à la pipette. Même dans ce cas il arrive que l'animal rende le lait par le nez.

Il faut évidemment tenir exactement compte des quantités effectivement ingérées et n'utiliser que des animaux auxquels on réussit à faire vraiment ingérer du lait, car il est à remarquer que certains sujets sont absolument réfractaires à ce genre d'alimentation. C'est à cela que nous attribuons essentiellement les grandes différences que l'on trouve dans les résultats qui ont été publiés.

Nous donnons, ci-dessous, des graphiques résumant nos essais pour la détermination des quantités de lait minima nécessaires au bon développement des cobayes. Nous voyons qu'un cobaye de 300 à $500 \mathrm{gr}$. se développe normalement avec $60 \mathrm{~cm}^{3}$ par jour de lait de femme, moyennant une augmentation de la dose de lait ingérée proportionnelle à l'augmentation de poids du sujet. Pour le lait de vache, on obtient le même résultat.

Il résulte, de ce qui précède, qu'un enfant de 5.000 à $6.000 \mathrm{gr}$, aurait besoin de 850 à $1.000 \mathrm{~cm}^{3}$ de lait maternel si les besoins spécifiques en vitamines " $\mathrm{C}$ " étaient les mêmes chez l'enfant et le cobaye. Ce n'est évidemment pas le cas. On a prétendu que les besoins de l'enfant en vitamines étaient environ cinq fois moindres que ceux du cobaye, mais ces essais ont probablement été faits avec du jus d'orange ou de citron. Il découle de nos propres essais, résumés dans le graphique I, que les 
Graphique I. - Lait de femme et lajt de vache Nourriture basale sans vitamine" C.

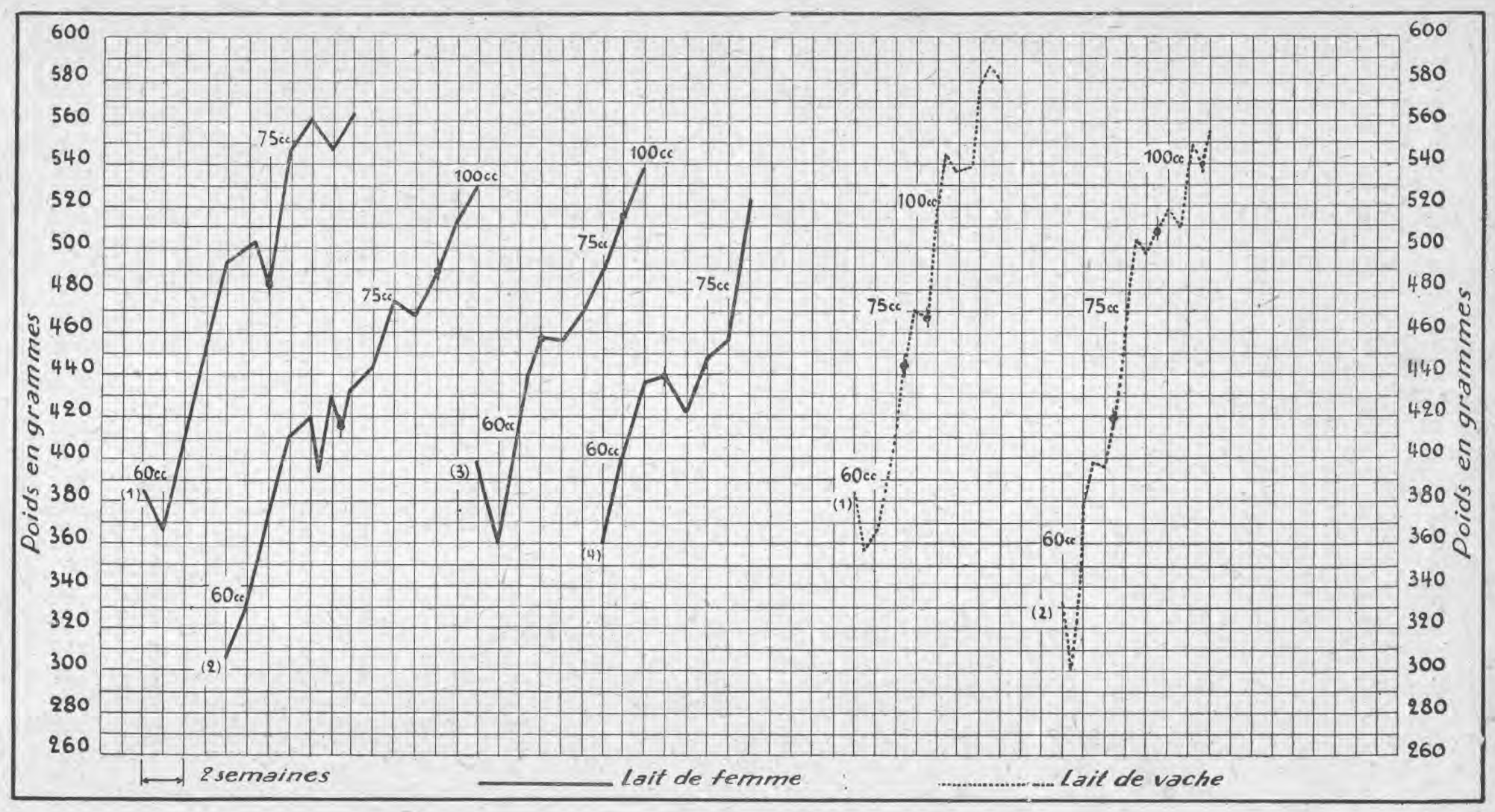


_ Graphique II. - Lait condensé sucré Nestlé. Nourriture basale sans vitamine C.

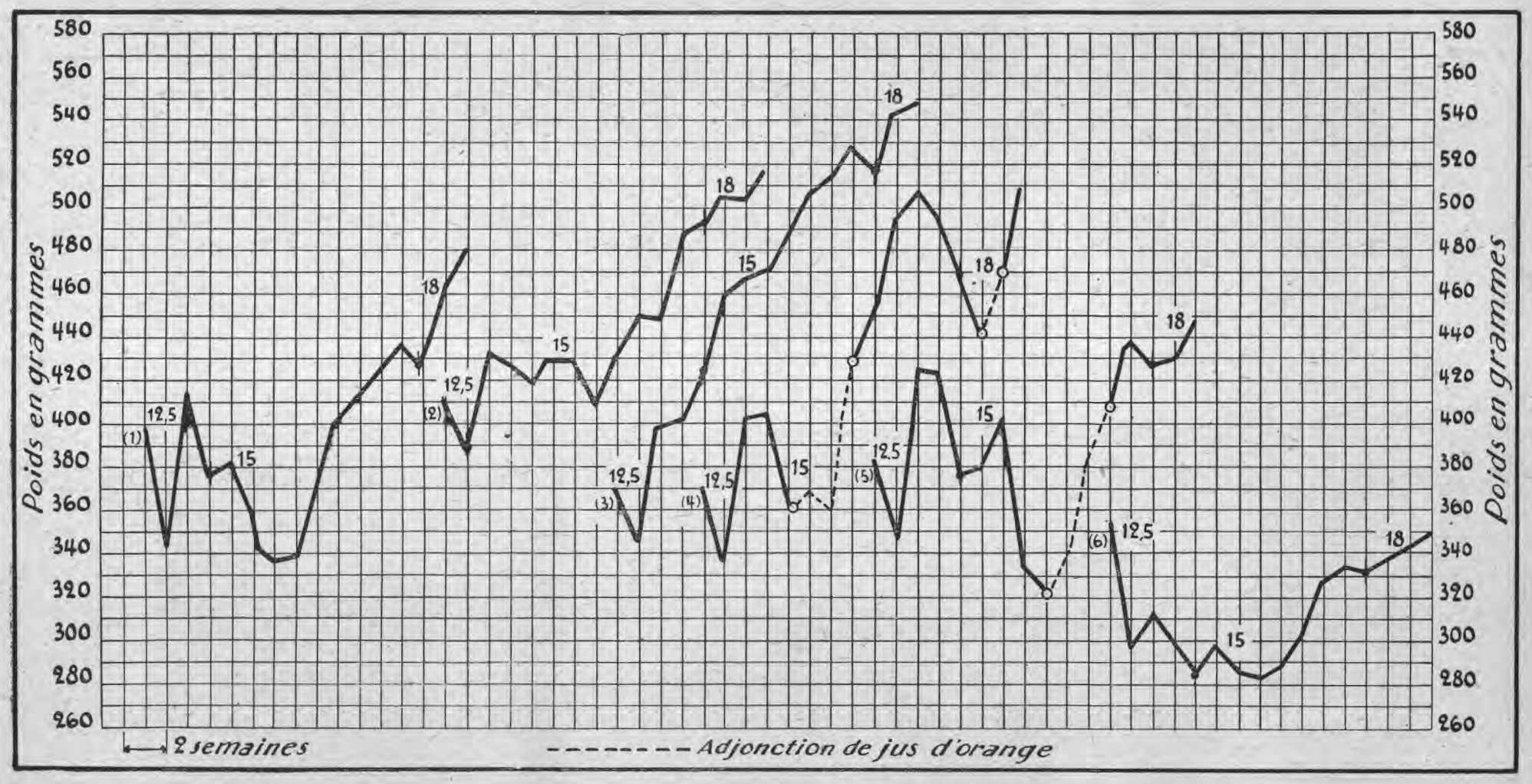


GraPHIQUE III. - Lait condensé sucré Nestlé. Nourriture basale sans vitamine C.

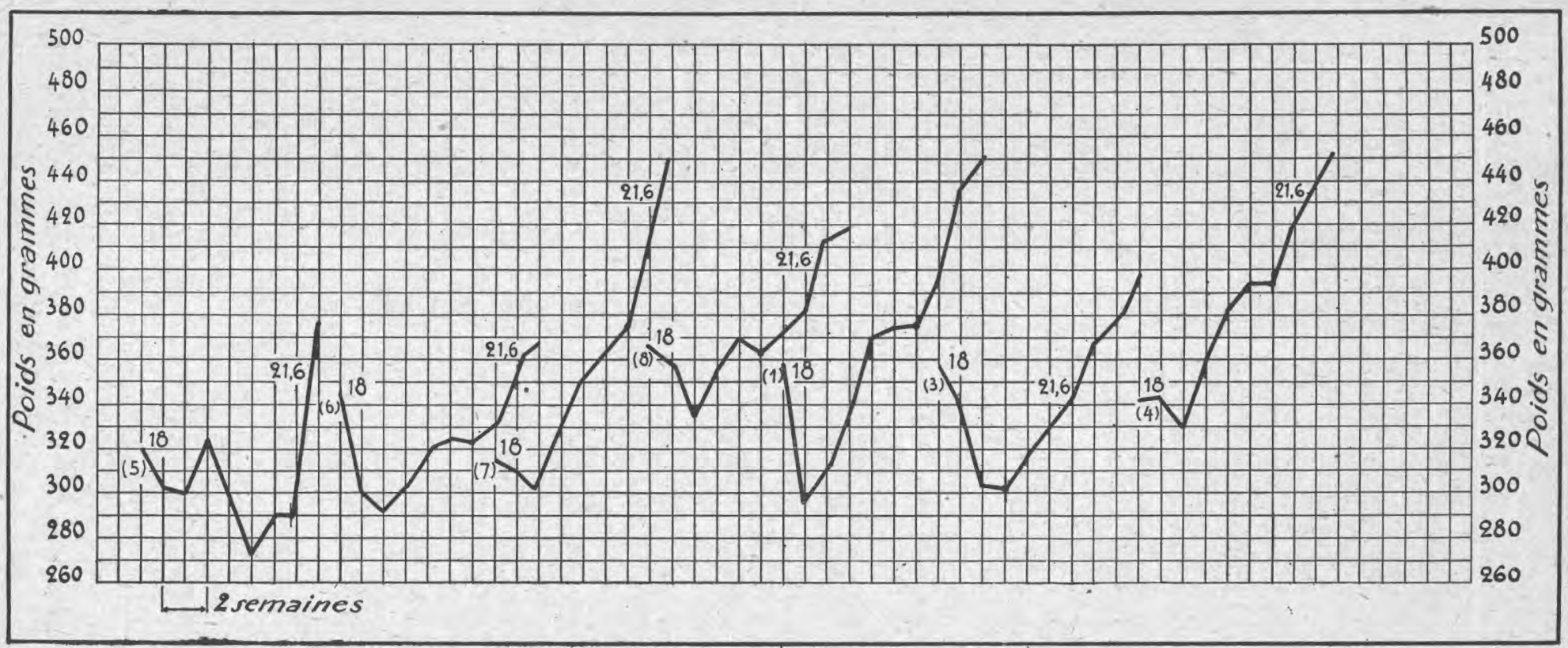

$\cdot$ 
besoins spésifiques de l'enfant en vitamines " $\mathrm{C}$ ) doivent être environ la moitié de ceux des cobayes, par unité de poids. Un coefficient de ce genre est de par sa nature même, approximatif.

\section{La Vitamine " $C$ " dans le lait concentré sucré}

Divers travaux ont déjà été publiés à ce sujet. LESNÉ et ses collaborateurs, notamment, ont démontré que la vitamine " $\mathrm{C}$ » dul ait frais est bien conservée dans le lait concentré sucré, mais qu'au bout de 15 mois son activité antiscorbutique commence à s'atténuer, et qu'après deux ans elle est devenue très faible. Il est à remarquer que, en pratique, les laits concentrés sucrés sont toujours consommés l'année même qui suit leur fabrication.

Lorsqu'on opère avec des cobayes, il faut se souvenir que ces animaux n'aiment pas le lait concentré sucré.

Comme ces animaux ne supportent pas bien de grandes quantitès de liquide, on est limité dans la dilution du lait concentré. Certains cobayes prennent ce produit assez facilement, sans trop de difficulté, tandis que d'autres le refusent absolument, et l'on est obligé d'abandonner les essais. Tci encore, on devra s'assurer des quantités réellement ingérées.

Nous avons effectué plusieurs séries d'essais, en faisant varier certains facteurs de l'une à l'autre, tels que l'âge du lait concentré ou sa provenance. Les graphiques ei-joints résument ces essais. Dans les deux premières séries (Graphiques II et III), et particulièrement dans la première, nous avons constaté que de petites quantités de lait concentré étaient suffisantes. Dans un cas, nous avons même noté un bon développement avec $15 \mathrm{gr}$, de lait concentré seulement par 24 heures, ce qui correspond à environ $40 \mathrm{~cm}^{3}$ de lait frais.

Dans la règle, il est cependant nécessaire d'administrer environ $18 \mathrm{gr}$. de lait concentré sucré par 24 heures, ce qui correspond à environ $50 \mathrm{~cm}^{3}$ de lait frais. Dans la $1^{\text {re }}$ série (Graphique II), le lait utilisé a vait été produit en automne et au commencement de l'hiver. Dans la $2^{\mathrm{e}}$ série, (Graphique III), il a fallu augmenter les doses jusqu'à 21,5 grammes par $24 \mathrm{~h}$. de lait concentré, ce qui correspond à $60 \mathrm{~cm}^{3}$ de lait frais. Ce lait a vait été produit au printemps et au commencement de l'été.

Les deux premières séries dont il vient d'être question ont été exécutées a vec du lait provenant de la même usine et constamment prélevé sur des lots de fabrication récente.

Examinons, maintenant, les résultats obtenus avec du lait de la même usine que pour les deux premières séries, mais provenant d'un mêne lot et dont l'âge augmentait donc automatiquement pendant l'essai, Malgré cela, on voit que les quantités de lait nécessaires restent sensiblement les mêmes que dans les deux premières séries. Toutefois, nous remarquons que l'âge du lait a une certaine influence sur les quantités ì donner, mais cette influence est si faible que pratiquement cela 
Graphique IV. - Lait condensé sucré Nestlé. Nourriture basale sans vitamine C.

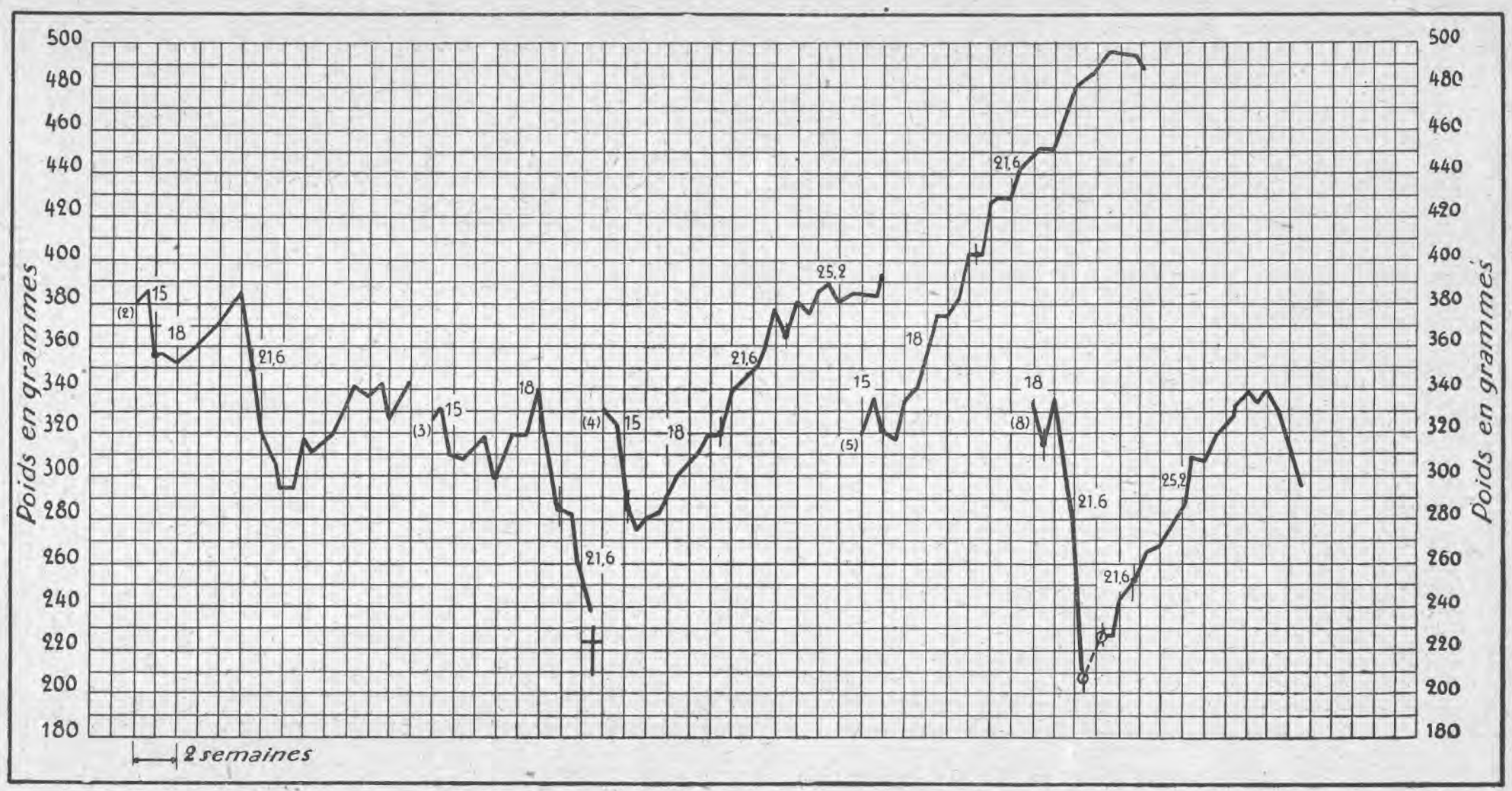


GraphIqUe V. - Lait condensé sucré Nestlé. Nourriture basale sans vitamine C

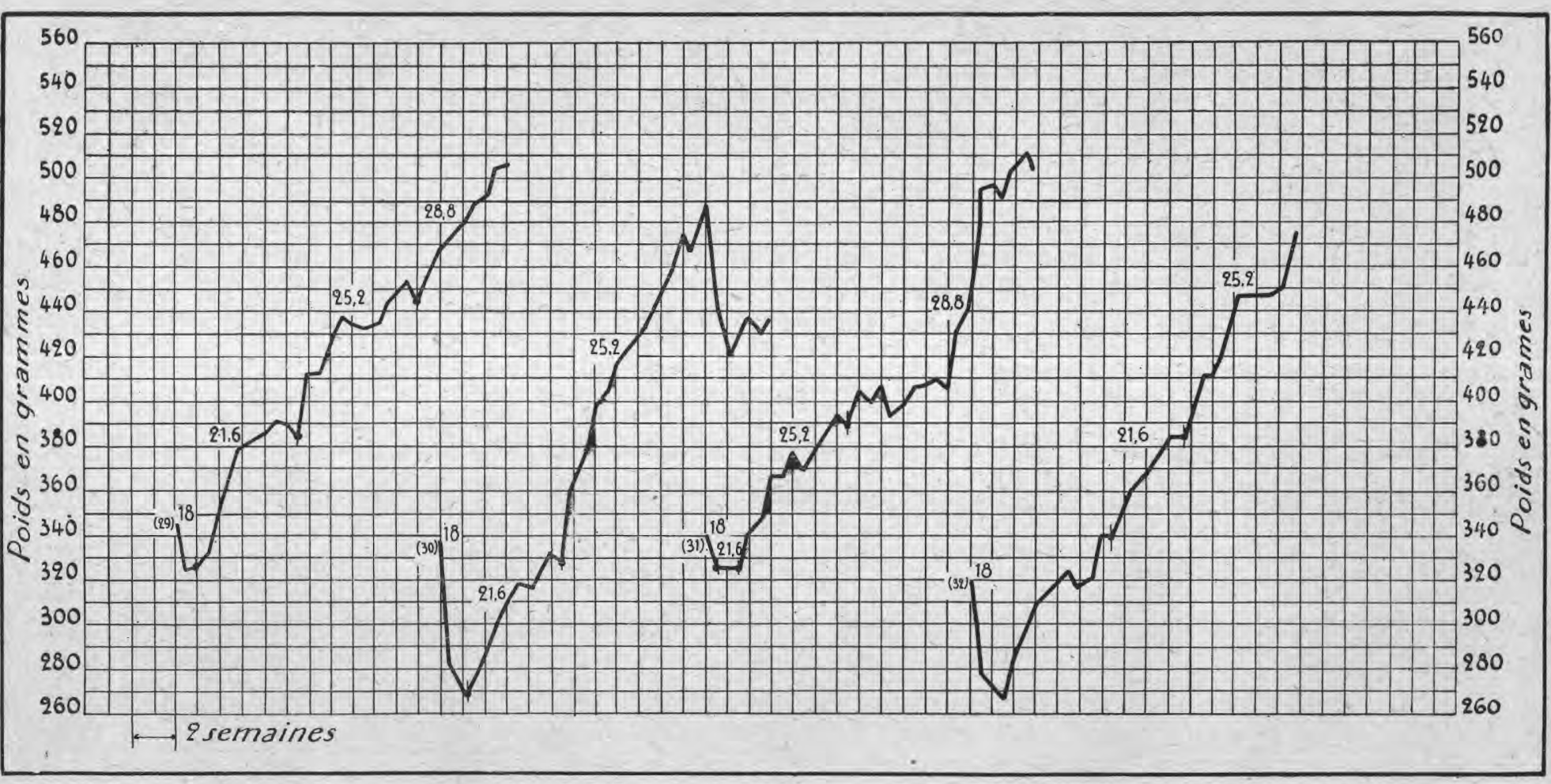


GraPHIQUE VI. - Lait condensé sucré Nestlé. Nourriture basale sans vitamine C.

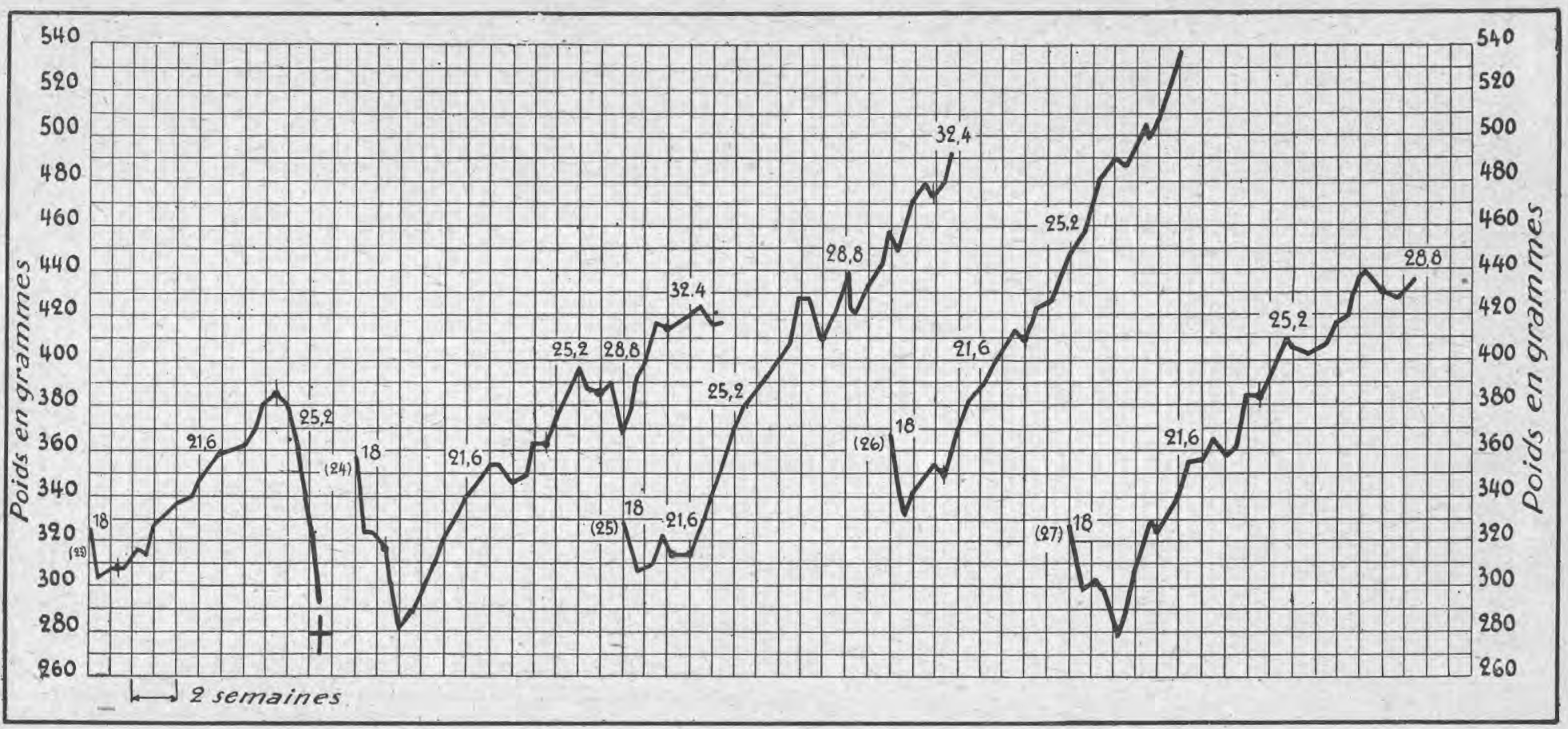


GrapHIQUe VII. - Lait condensé sucré Nestlé. Nourriture basale sans vitamine C.

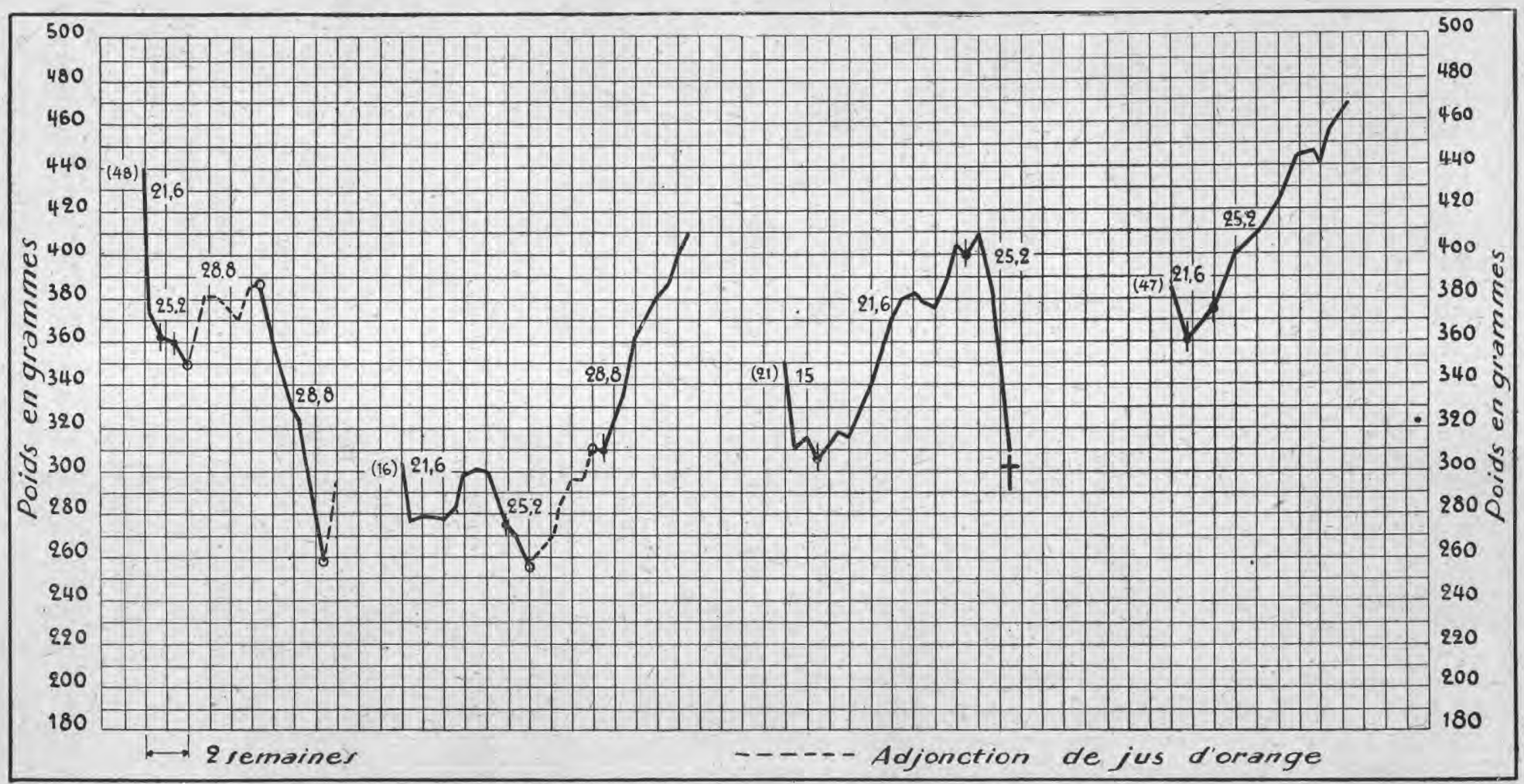


n'a pas d'importance. Nous avons eu beaucoup de peine à faire accepter le lait par les animaux et l'essai a dô être abandonné pour plusieurs d'entre eux. L'un d'eux, qui prenait facilement le lait, s'est développé normalement, avec des quantités à peu près identiques à celles que nous avions employées dans les deux premières séries.

$A u$ moment où l'essai a été interrompu, le lait était vieux de 6 mois.

Les Graphiques V et VI resument des essais effectués avee du lait sucré provenant d'un autre pays que les trois premières séries. Les autres conaitions étaient ies mêmes que celles du Graphique IV.

Nous voyons qu'il a fallu augmenter très légèrement la ration de lait entre les séries représentées par les Graphiques V et VI. La seule diflérence entre les deux séries existait dans la pasteurisation. On voit que cela n'a pas eu d'influence sur la vitamine " $\mathrm{C}$ ".

Les graphiques ci-dessous donnent le résultat obtenu avee un lait concentré d'un autre district, mais du même pays que dans les Graphiques V et VI. Nous voyons que, dans le cas des Graphiques VII, il a fallu donner davantage de lait pour assurer un développement normal aux sujets. Nous avons eu beaucoup de difficulté, dans cette série, pour assurer l'alimentation aux animaux. Mais, même dans les eas où l'alimentation a pu être assurée normalement, il a fallu da vantage de lait que'dans les cas précédents.

Les Graphiques VIII et IX résument des essais dans lerquels la quantité de lait n'a pas été sensiblement plus grande que dans 1 irucicis précédents. Il s'y trouve, cependant, certains cas où il a fallu forcer la dose quelque peu. Le lait utilisé pour les séries VIII et IX provenait d'un pays autre que celui qui a été utilisé dans les séries précédentes.

Nous insistons encore sur les difficultés que l'on rencontre dans l'emploi des cobayes au cours d'essais portant sur le lait concentré, du fait de leur aversion pour cet aliment. Comme il y a, à ce point de vue, des différences individuelles très grandes chez les cokayes, il fant, pour obtenir dès résultats utilisables, precéder à des efsais sur des grcupes d'animaux.

Nous avons constaté que si, pendant un certain temps, les quantités ingérées par les sujets ne permettent pas d'assurer leur développement normal, ils ne peuvent le reprendre, même si l'on augmente la quantité de lait. Il est aloirs nécessaire de leur donner du jus d'orange pour les remonter avant de continuer l'essai.

Il est intéressant de constater que, fréquemment, moyennant ce traitement, les animaux continuent leur développement, même avec les quantités de lait qui étaient insuffisantes auparavant. On dirait qu'il leur a suffi de prendre un élan.

On pourrait penser, également, à la théorie qui veut que les toxines de l'organisme soient chassées par les vitamines. Sous cet angle, on dirait 
GraPHIQUe VII. - Lait condensé sucré Nestlé. Nourriture basale sans vitamine C.

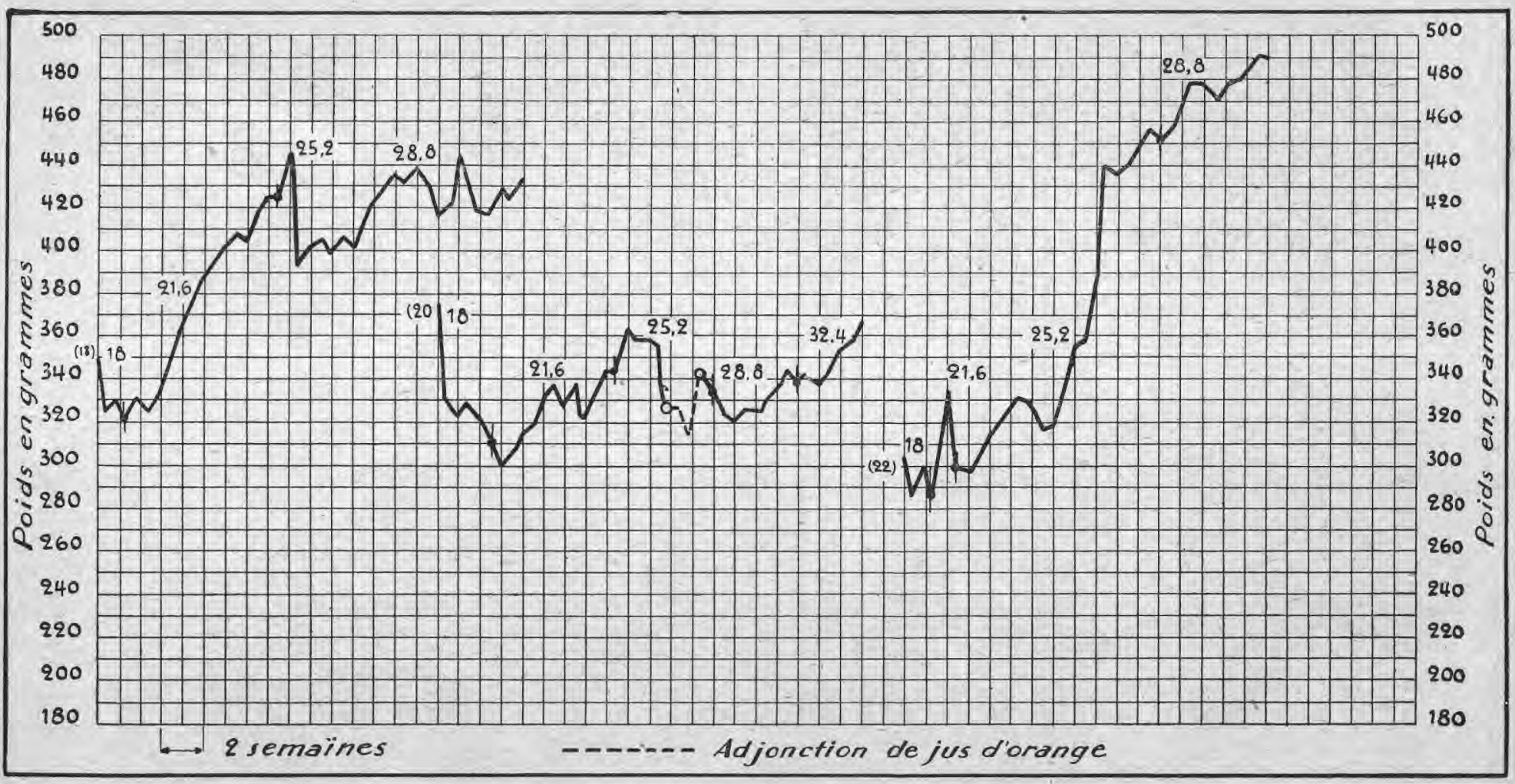


GrapHQUe VIII. - Lait condensé sucré Nestlé. Nourriture kasale sans vitamine C.

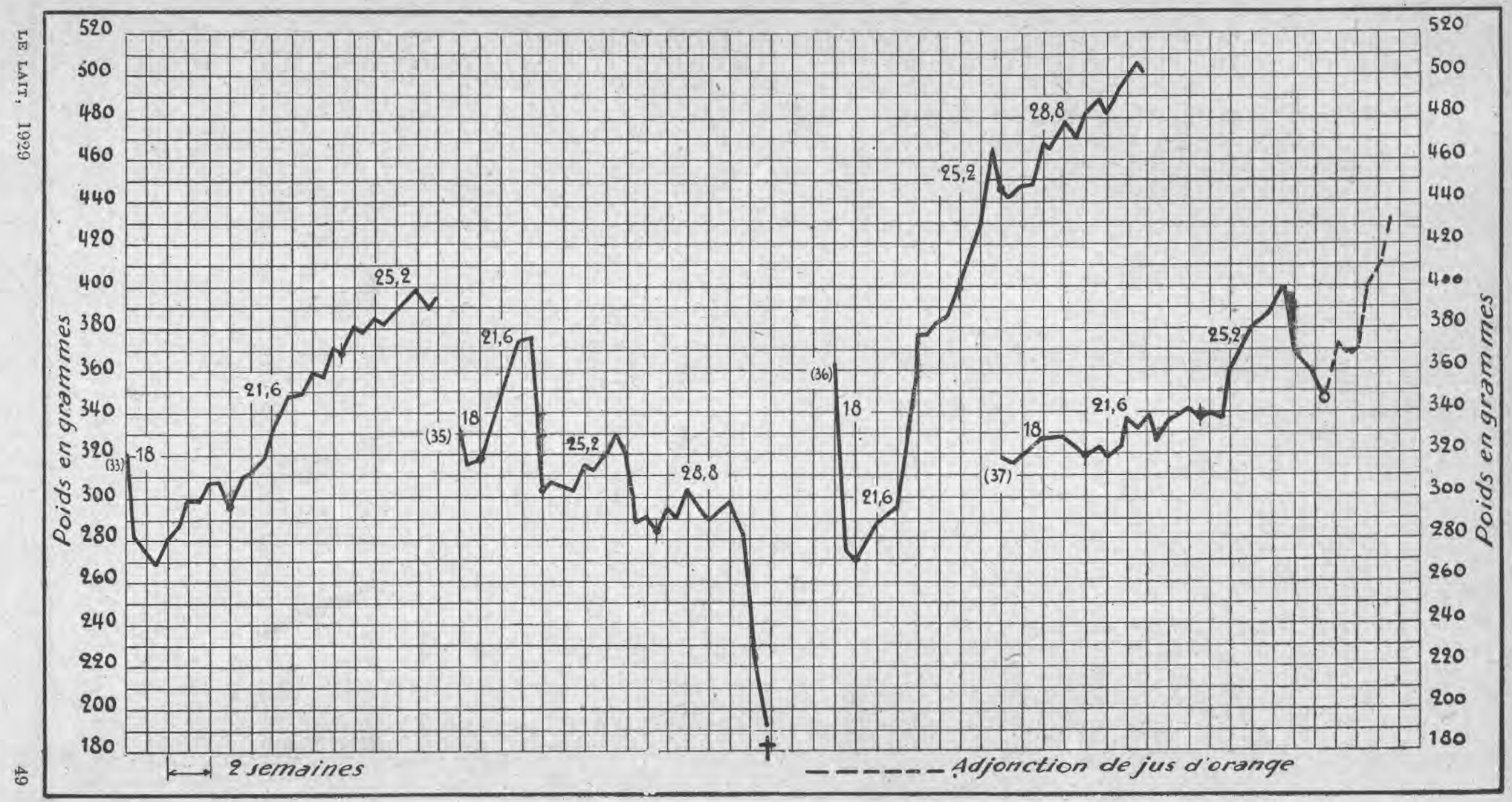


Graphique IX. - Lait condensé sucre Nestlé. Nourriture basale sans vitamine C.

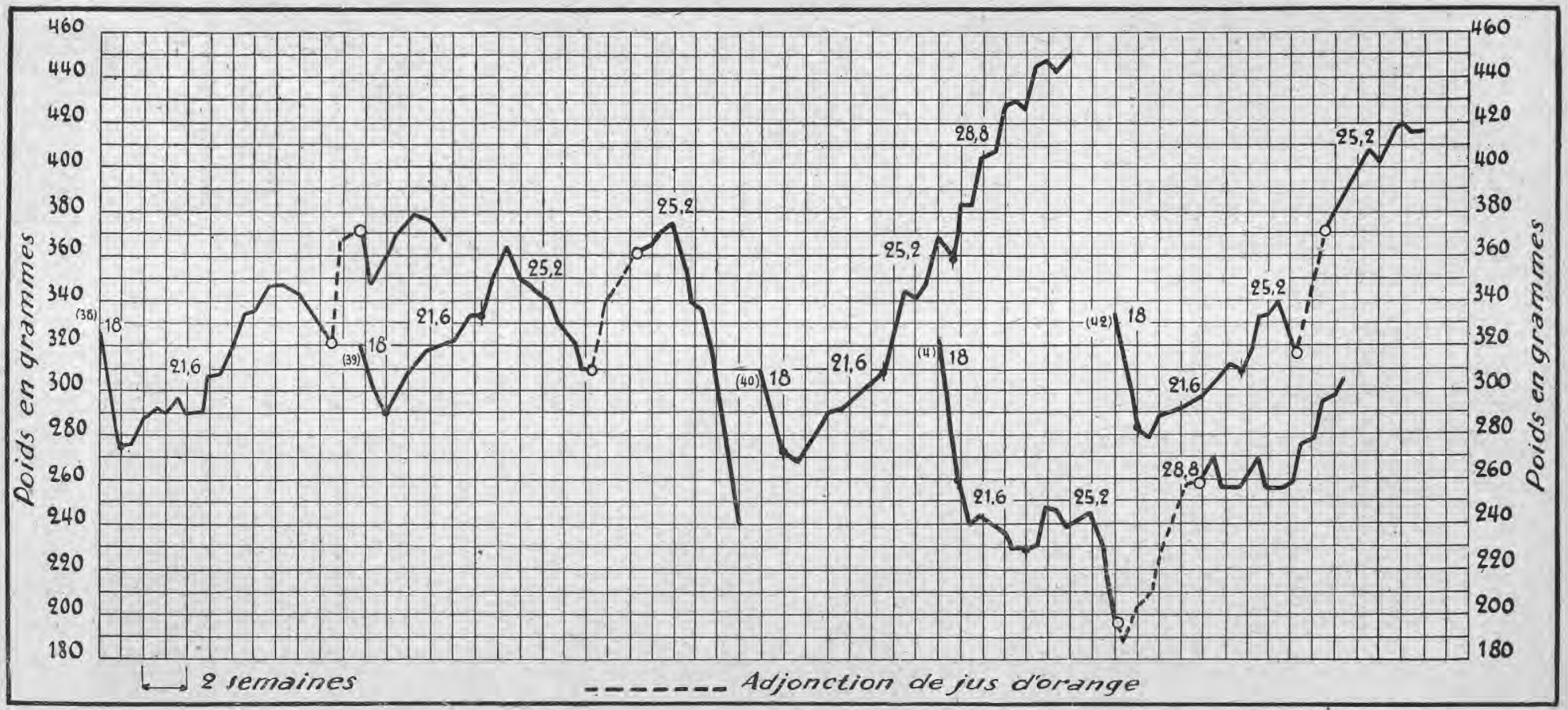


alors qu'il s'est produit une açcumulation de toxines devant être chassées afin d'assurer le retour au développement normal.

D'autres auteurs déjà ont constaté que l'activité du principe antiscorbutique du lait diminue avec l'âge. C'est ce que nos essais ont confirmé. Cependant, nous pouvons également confirmer que le vieillissement ne se fait pas sentir d'une façon appréciable tant que le lait n'est pas vieux au sens commercial du mot, pourvu que les methodes de fabrication employées soient réglées en conséquence.

Nous avons pu constater, notamment, qu'une pasteurisation convenable, telle qu'elle est réalisée dans les usines Nestlé, reste sans influence sur la vitamine "C ".

Nous avons vu plus haut, que les besoins spécifiques des enfants en vitamines " $C$ " pouvaient être évalués à environ la moitié de ceux des cobayes. Nous avons vu également, au début, que, pour des cobayes pesant de 300 à 500 grammes, il faut de 18 à 28 grammes de lait concentré par jour pour couvrir leurs besoins en vitamines " $\mathrm{C}$ ", soit en moyenne 23 gr., ce qui correspond à $65 \mathrm{~cm}^{3}$ de lait frais, saccharose non compris.

En tenant compte de ces éléments, on arrive done à la conclusion que, pour un enfant de 5 à 6.000 gr., il faut compter un minimum de $650 \mathrm{~cm}^{3}$ par 24 heures, environ, de lait frais, pour couvrir les besoins en vitamines, ce qui correspond à environ $230 \mathrm{gr}$. de lait concentré par $24 \mathrm{~h}$., cela si les besoins étaient les mêmes. Comme la moitié seulement est nésessaire, nous arrivons à environ $115 \mathrm{gr}$. seulement de lait concentré.

Comme, pour tenir compte des besoins énergétiques de l'enfant, les quantités de lait frais ou de lait concentré sucré effectivement administrées par 24 heures sont très supérieures aux chiffres théoriques, ealculés sur la base du besoin en vitamines " $C$ ", on voit que, au point de vue antiscorbutique, l'alimentation des enfants au lait concentré sucré couvre, avec un coefficient de sécurité considérable, les besoins en vitamines " $\mathrm{C}$ " du nourrisson.

C'est le résultat auquel est arrivé également le $\mathrm{D}^{\mathrm{r}}$ Hottinger, de la elinique infantile universitaire de Bâle, selon sa publication dans le Journal hebdomadaire de Médecine, № 5, 1929.

Comme la vitamine " $\mathrm{C}$ " est la plus fragile, il est clair que les autres vitamines du lait frais se retrouvent intactes dans le lait concentré sucré. C'est ce qu'ont confirmé de nombreuses séries d'essais, que nous avons effectués pour chacune des autres vitamines prises séparément. Mais les faits résumés dans le présent article suffisent, pensons-nous, à en faire la preuve indirecte.

(A suivre.) 